\title{
DEL CÓLICO MISERERE A LA APENDICECTOMÍA LAPAROSCÓPICA*
}

\author{
Dr. Osvaldo Llanos L. ${ }^{1}$ \\ 1 Departamento de Cirugía Digestiva. Facultad de Medicina, Pontificia Universidad Católica de Chile. Santiago. \\ Chile.
}

\begin{abstract}
From colico miserere to laparoscopic appendectomy

Colico miserere is an old expression used since the $17 \mathrm{~h}$ century to denominate different acute abdominal inflammatory diseases, as intestinal obstruction, acute appendicitis and others, all of them with difficulties to differentiate among each others and with high mortality rate at that time. Some of the history of these diseases is briefly reviewed. Historical arguments to consider acute appendicitis as a single nosologic entity and its surgical treatment are discussed.
\end{abstract}

Key words: Colico miserere, appendicitis, intestinal obstruction.

\section{Resumen}

El término cólico miserere se usó desde el siglo XVII para los diferentes cuadros abdominales agudos, como la obstrucción intestinal y la apendicitis aguda, entre otros, de difícil diagnóstico diferencial y alta mortalidad en esa época. Se revisa, en breve, parte de la historia del conocimiento y tratamiento de ambas enfermedades desde la era antes de Cristo. Se enfatiza en las dificultades para considerar a la apendicitis aguda como una entidad nosológica aparte y para definir su tratamiento quirúrgico.

Palabras clave: Cólico miserere, apendicitis, obstrucción intestinal.

La expresión cólico miserere, o cólico del miserere, o miserere mei, se usó desde el S XVII hasta fines del S XVIII para denominar primero un cuadro abdominal agudo compatible, hoy día, con una obstrucción intestinal, de distintas y desconocidas causas y que irremediablemente llevaba al enfermo a la muerte. También se usó para denominar a la apendicitis aguda, la que muchas veces se perforaba, provocaba la peritonitis y la muerte. La mayoría de las veces ambas enfermedades se confundían y el diagnóstico se aclaraba, a veces sólo con la autopsia, procedimiento que se utilizó en forma sistemática desde la misma época, el siglo XVII-XVIII. De esta manera, es comprensible la confusión diagnóstica que existía en esa época, entre la obstrucción intestinal y la apendicitis aguda y probablemente más de algún otro cuadro abdominal agudo, de otra etiología ${ }^{1}$.

\footnotetext{
No hay conflictos de interés.

Correspondencia: Dr. Osvaldo Llanos L.

ollanos@puc.cl
}

*Recibido el 27 de diciembre de 2013 y aceptado para publicación el 22 de enero de 2014. 
El cólico miserere se describía como un dolor abdominal muy intenso, vómitos fecaloídeos y muerte $^{2,3}$. La aparición de estas manifestaciones, junto al rápido e inevitable fallecimiento del enfermo, hacía que los vecinos y conocidos del enfermo se reunieran a su alrededor a cantar el Miserere para pedir piedad a Dios, como una plegaria para la curación del paciente. Dicho canto era también característico en las misas de difuntos, de manera que se establecía una rápida relación entre la aparición de la enfermedad, la muerte y los actos religiosos del sepelio. La palabra Miserere en latín significa apiádate, ten piedad, y este canto del Miserere o también llamado Miserere mei Deus (Oh Señor, ten piedad de mí), resultó de una obra coral de Gregorio Allegri compuesta en 1630. Allegri, uno de los pioneros del barroco italiano, era músico del Vaticano y compuso el Miserere a solicitud expresa del Papa Urbano VIII. Esta composición musical, para dos coros, fue acompañada del Salmo 51 y se dispuso que se cantara, en forma exclusiva, en los maitines del Miércoles y Viernes Santos. En un ambiente de estricto secreto, se mantuvo oculta, bajo pena de excomunión, para su uso exclusivo en Semana Santa, hasta aparentemente 1770, año en que el secreto fue vulnerado por Mozart, quien lo retuvo en su mente genial después de una visita al Vaticano ${ }^{1}$.

La obstrucción intestinal, también llamada "pasión ilíaca", fue descrita antes de Cristo y generalmente estaba en relación a una hernia estrangulada. A pesar de que el curso era irremediablemente mortal, algunas maniobras terapéuticas están descritas ya por Hipócrates, quien usaba, entre otras, enemas de distinto tipo e insuflación anal de aire. Hipócrates, además, fue el primer médico que acuñó la palabra "ileus". En la medicina de la antigua Grecia, también está descrito que Praxágoras, influyente médico de la época, 300 años aC, realizaba una incisión sobre la hernia estrangulada en un intento por tratar la obstrucción y la vida del enfermo, la mayoría de las veces con los resultados imaginables. Por otra parte, en la Medicina india antigua de 6 a 8 siglos aC, los famosos escritos en sánscrito, como el Susrhuta Samhita, simplemente declaraban que estos cuadros como la obstrucción intestinal eran enfermedades incurables.

Siglos después comenzaron los intentos de tratamiento quirúrgico, la mayoría de ellos fallidos, como los de la escuela de Saliceto, en el siglo XIII, quien, al menos tuvo el mérito de recuperar el uso del bisturí, después que éste había sido casi desplazado por el cauterio, por la escuela árabe ${ }^{4,5}$.

Entre las medidas terapéuticas, algunas casi anecdóticas, descritas para la obstrucción intestinal, destaca, por ejemplo la aplicación de corriente farádica, recomendada por el francés Duchenne (1855) y por sus seguidores Beard y Rockwell, quienes en 1871 escribieron un libro sobre "Electroterapia". Así también, son clásicas las maniobras terapéuticas para la obstrucción intestinal, consistentes en la ingestión de metales por los enfermos, bolas de plomo y mercurio entre los más usados. Ya en el siglo XVI, Marianus Sanctus utilizaba esta medida, la que fue reactualizada por Leichstestern hacia 1876. A esta ingesta de metales se agregaba con frecuencia el uso de cambios de posición del enfermo (Hutchinson, 1878), con el propósito de facilitar el avance de la carga metálica, para promover así la desobstrucción del intestino. Naturalmente que la mayoría de los enfermos terminaban con la muerte por ruptura intestinal. Astley Cooper, el famoso precursor de la cirugía de las hernias, describió en 1804, la utilidad de la posición invertida del paciente, acompañada de maniobras de taxis para reducir una hernia atascada ${ }^{5}$.

A partir del Siglo XVI, junto con el uso de las medidas descritas, comienza también el tratamiento quirúrgico más sistemático de la obstrucción intestinal, con Ambroise Paré, en París, considerado el padre de la cirugía moderna (1510-1592). Luego, dos siglos más tarde, en la cirugía del Romanticismo, destacaron Astley Cooper (1768-1841) en Inglaterra y Auguste Nelaton (1807-1873) en París, entre otros ${ }^{3}$. Naturalmente que, los malos resultados iniciales de la cirugía, mantuvieron por largo tiempo la controversia entre el tratamiento operatorio y las medidas menos agresivas, como enemas de todo tipo, sondas por vía oral y rectal, calor abdominal, el reposo y el uso de opio, cuyo uso masivo en las enfermedades inflamatorias abdominales fue introducido por Stokes en Dublín en $1838^{5}$, en contradicción con otros que llegaban a indicar ejercicios físicos e incluso las cabalgatas a caballo.

Recién hacia fines del siglo XVIII se comenzó a ver un cierto progreso en el tratamiento quirúrgico con el comienzo de las resecciones intestinales y las primeras ostomías, para continuar, a comienzos del siglo XIX con las primeras técnicas de anastomosis intestinales.

La apendicitis aguda es la otra enfermedad que se incluía en el término Colico Miserere, enfermedad tan frecuente y mal conocida hasta fines del siglo XIX, de altísima mortalidad hasta esa época, de diagnóstico a veces difícil, hasta hoy, a pesar de los progresos en las imágenes, con muy bajo riesgo actual pero todavía con potenciales graves complicaciones y eventual mortalidad, en especial en casos de diagnóstico tardío. A pesar de que, en la actualidad, el arte médico pareciera tener menos trascendencia y haber perdido terreno frente a la cada vez más tecnificada Medicina moderna, se podría decir que en enfermedades como la apendicitis aguda, se hace más necesario su sabio uso. A través 
de la historia, la apendicitis aguda ha sido motivo de varias controversias por diferentes motivos. La primera fue la dificultad que tuvo para establecerse como una entidad nosológica propia, diferente de otras afecciones abdominales agudas, englobadas todas en los términos colico miserere, tiflitis o pasión ilíaca derecha.

Al buscar en la historia, parece que los primeros indicios de apendicitis agudas están en las momias de Asuán, que datan de 3.000 años $\mathrm{aC}$, en las que se ha encontrado signos compatibles con peritonitis en la fosa ilíaca derecha que podrían corresponder a perforaciones apendiculares. En la Grecia antigua, Hipócrates $(450 \mathrm{aC})$ describe también procesos compatibles con perforaciones apendiculares y peritonitis en la fosa ilíaca derecha ${ }^{5,6}$. Varios personajes de la historia tienen relatos compatibles, como por ejemplo Herodes Agripa, quien falleció en el año 44 $\mathrm{aC}$, con un cuadro, en relato de Flavio Josefo, muy sugerente de una apendicitis. Maquiavelo también, quien falleció en 1527, según algunos, "de pena", después de la pérdida del poder, describe dolores abdominales muy sugerentes de una apendicitis. Erasmo de Rotterdam en 1530, también describió dolores en fosa ilíaca derecha compatibles con una apendicitis, los que regresaron y aparentemente no se complicó ${ }^{\text {. }}$.

Respecto al conocimiento anatómico del apéndice, son notables las ilustraciones de Leonardo Da Vinci, quien lo dibujó en sus célebres dibujos anatómicos en 1492. Da Vinci denominó al apéndice con el término "orecchio" o la oreja del intestino $0^{7,8}$. Sin embargo, el mérito de la primera descripción anatómica del apéndice, corresponde al anatomista de Bolonia, Berengario da Carpi, quien lo describió en $1521^{9}$. Posteriormente, en 1554, Jean Fernel, famoso filósofo, matemático y médico, a quien se ha llamado el Galeno francés, hizo la primera descripción de una apendicitis en una autopsia ${ }^{10}$. Vesalio también ilustró el apéndice en su obra "De Humani Corporis Fabrica" en 1543 y J. B. Morgagni describió también más tarde, con claridad su anatomía en 1719. Con posterioridad, se conocen una serie de descripciones de apendicitis aguda y en especial de abscesos en fosa ilíaca derecha secundarios a ella. Entre las más famosas, se destacan la de Lorenz Heister en 1711, quien describió un absceso junto a un apéndice negro en la autopsia de un criminal ejecutado, la de Mestivier en Francia en 1759, la de John Hunter en 1767 y la de James Parkinson quien describió un apéndice perforado con un fecalito en la autopsia de un niño de 5 años en 1812. En la Real Academia de Medicina de París, Louyer-Villermany presentó en 1824 la autopsia de dos hombres jóvenes con el apéndice gangrenado y, en 1827, Francois Melier, sugirió por primera vez la apendicectomía después de presentar otros 6 casos en autopsias ${ }^{8}$.

El concepto de una inflamación aguda en la fosa ilíaca derecha originada en el apéndice, se vio fuertemente afectado por la idea que toda esta inflamación en la fosa ilíaca derecha provenía básicamente del ciego, idea preconizada principalmente por el influyente barón de Dupuytren (1777-1835), quien acuñó el término de tiflitis para todos estos proce$\operatorname{sos}^{7}$. Esta interpretación fue apoyada por numerosos seguidores, entre los que destacan Goldbeck y Alberts (1830), y lo único que se obtuvo fue retrasar el tratamiento quirúrgico de la apendicitis aguda. En forma trágica, la muerte del renombrado cirujano norteamericano Ephraim McDowell, en 1830, a los 58 años por una peritonitis apendicular, remeció el ambiente médico y estimuló a los líderes de la época a insistir en el diagnóstico de la apendicitis aguda. Es así como en 1839, Bright y Addison, del Guy's Hospital, en Inglaterra, describieron los síntomas de la apendicitis aguda y lo publicaron en los "Reports" del Guy's Hospital, famosa y pionera publicación médica, aún cuando no mencionaron el tratamiento ${ }^{7}$.

En relación al tratamiento quirúrgico, la primera operación sobre el apéndice fue realizada en 1735 por Claudius Amyand, cirujano londinense, hugonote refugiado, quien intervino, sin anestesia, a un niño de 11 años con un apéndice perforado por un alfiler en una hernia inguinal complicada. De ahí quedó denominada como hernia de Amyand la hernia inguinal con el apéndice en su interior ${ }^{11-13}$. Más de un siglo después aparecieron comunicaciones de otras intervenciones, fundamentalmente drenajes de abscesos apendiculares. Así, en 1848, Hancock en Londres drenó un absceso apendicular en una puérpera de 30 años y en 1867, Parker en Nueva York presentó varios drenajes en enfermos con un absceso apendicular. La primera apendicectomía propiamente tal, por apendicitis aguda, aparentemente fue realizada por el cirujano escocés Lawson Tait en 1880 en una mujer de 17 años. Tait destaca en la historia de la Cirugía por ser también el primero en realizar una colecistostomía, la primera salpingectomía y la primera ooforectomía. Curiosamente, Tait era contrario al Listerismo y creía que el gas carbónico era peligroso. Junto a Marion Sims son considerados los padres de la ginecología. Luego, de 1883 a 1889 se comunicaron varias apendicectomías, por cirujanos como Groves, Mikulickz, Kronlein (en Zurich), Charter-Symonds en Londres, Hall en Nueva York, Sands, McBurney y Morton en los Estados Unidos, no todas, por supuesto, con sobrevida del paciente.

La historia del diagnóstico y de la indicación quirúrgica de la apendicitis aguda fue marcada en forma trascendente por la presentación del doctor Reginald Fitz, profesor de Anatomía Patológica de la Universidad de Harvard, en 1886, ante la Asso- 
ciation of American Physicians, en Washington. El doctor Fitz mostró los resultados de autopsia de 257 enfermos fallecidos con apendicitis aguda perforada, 209 de ellos catalogados como tiflitis. En su trabajo "Perforating inflammation of the vermiform appendix: with special reference to its early diagnosis and treatment" Fitz acuñó el término "apendicitis" y recomendó la apendicectomía precoz, terminando con la polémica con el diagnóstico de tiflitis y su tratamiento médico ${ }^{13,14}$. Después de este hito, comenzó con menos dudas, el tratamiento quirúrgico de la apendicitis. Así, Thomas G. Morton en Filadelfia en 1887, operó un paciente con diagnóstico previo de apendicitis aguda perforada, le realizó una apendicectomía y drenaje con sobrevida del enfermo ${ }^{7}$. John Benjamin Murphy, otro prócer clásico en la historia de la cirugía, describió la cronología de los síntomas de la apendicitis aguda y presentó en 1889 , ante la Chicago Medical Society, 8 casos de apendicectomía por apendicitis aguda. En 1904 una nueva presentación ya contaba con 2.000 casos $^{10}$.

Charles McBurney, otro pionero en la historia de la cirugía, con múltiples contribuciones a ella, estableció guías para el diagnóstico y el tratamiento de la apendicitis y son famosas sus descripciones sobre el punto doloroso en 1889, y sobre la incisión en 1894, que llevan su nombre ${ }^{15}$. McBurney fue también un impulsor de la técnica estéril y uno de los precursores, junto a Halsted, del uso de los guantes quirúrgicos ${ }^{16}$. Como anécdota, el doctor Lewis Mc Arthur en 1894, describió en Chicago, la misma incisión y la misma técnica que McBurney para la apendicectomía, pero su publicación fue atrasada. McBurney lo hizo ver en esa reunión en Chicago y le concedió todo el crédito a Mc Arthur, sin embargo, la historia le dio la fama a McBurney ${ }^{10}$. El francés G. Dieulafoy, quien, en 1897, ante la Academia de Medicina de París enfatizó que el tratamiento médico estaba destinado al fracaso y que el único tratamiento racional era el quirúrgico ${ }^{12}$, describió también la triada característica de esta enfermedad: dolor, defensa muscular e hiperestesia cutánea (1896). Por su parte, Albert Ochsner en 1902, publicó un manual sobre el tratamiento quirúrgico de la peritonitis apendicular, comunicando 1.000 casos, pero reactivando la controversia al proponer el tratamiento médico en los casos en que el proceso llevase más de 2 días $^{8}$. Ochsner proponía un tratamiento con régimen cero (nada por boca), lavados gástricos frecuentes y enemas nutrientes que permitiría que la peritonitis se localizara y luego se podría operar en forma más segura. En ese año 1902, un hecho conocido en el ambiente médico de la época, casi anecdótico, pero que apoyó también al tratamiento quirúrgico, fue la operación de Eduardo VII, sucesor de la reina Victoria, de una apendicitis aguda, días antes de su coronación. El cirujano designado para tratarlo fue Sir Frederik Treves, quien había operado la primera apendicitis en Inglaterra en 1888. Treves era un entusiasta defensor del tratamiento quirúrgico de estos cuadros abdominales agudos (obstrucción intestinal, peritiflitis, apendicitis aguda), debió convencer al rey, con el apoyo de Lister, para que se operara, ante su negativa inicial a hacerlo porque significaba postergar su coronación. Finalmente, el príncipe, después de varios días, aceptó la intervención y Treves le drenó un absceso apendicular. La coronación se realizó un par de semanas después ${ }^{17}$.

Siguiendo en el terreno anecdótico, la apendicectomía es una de las pocas operaciones que se incluyen dentro de los posibles ejemplos de auto cirugía, es decir, una intervención practicada por el cirujano sobre sí mismo. Aparentemente el primer cirujano que se extirpó su propio apéndice, con anestesia local, fue el doctor Evan O. Kane, jefe del Kane Summit Hospital, en EEUU, en $1921^{7}$. Kane realizó su autointervención para demostrar que era posible hacerla con anestesia local y evitar los riesgos del éter. Este mismo cirujano, tiempo después, en 1926, se operó también de una hernia inguinal. Posteriormente, se han comunicado al menos otros 3 cirujanos que se han autooperado el apéndice ${ }^{6}$.

En Chile la primera operación descrita sobre un absceso en la fosa ilíaca derecha fue realizada por el Profesor Manuel Barros Borgoño en 1890. En tanto que la primera operación por apendicitis aguda fue practicada por el doctor Olof Page en Valparaíso en 1894 quien realizó sólo un drenaje y días después tuvo que reoperar al paciente por un ileo (Rev Med Chile 1894;22:457-60). En estas dos primeras operaciones no se extirpó el apéndice y efectivamente la primera apendicectomía por apendicitis aguda fue realizada por el Profesor Ventura Carvallo Elizalde en $1896^{18}$. El doctor (alumno interno) Germán Aliaga Larrea hizo su tesis de grado sobre estos primeros casos titulada "Sobre apendicitis. Estudio hecho con ocasión de las primeras resecciones del apéndice vermicular practicadas en Chile", 1886. En el describe 3 casos, los dos primeros operados por el profesor Ventura Carvallo y el tercero por el profesor Raimundo Charlín.

Entre 1888 y 1889 continuaron los debates terapéuticos en la Sociedad Médica de Santiago sobre el tratamiento de la apendicitis aguda, con opiniones divididas. Hasta el año 1901, en que el profesor Ventura Carvallo, presentó 51 operaciones de apendicitis, concluyendo en forma categórica que el único tratamiento para esta enfermedad era el quirúrgico $^{18}$. Por su parte, desde Londres, en 1893, Lucas Sierra escribía para la Revista Médica de Chile el artículo titulado "La apendicitis consideradas desde el punto de vista quirúrgico", entre otras de sus 
contribuciones, como "La apendicitis y sus complicaciones, "La infección biliar", "La revolución de la Medicina en el siglo XIX". El distinguido pionero de la cirugía chilena afirmó que "La apendicitis, a pesar de todos los enigmas que encierra todavía, es la enfermedad histórica más importante que yo conozca", frase que con toda probabilidad mantiene hoy algo de su vigencia.

Posteriormente, viene todo el desarrollo de la cirugía moderna y contemporánea, con los progresos médicos y científicos del siglo pasado entre los que destacan los antibióticos, la nutrición parenteral, las medidas de soporte del enfermo crítico, que han contribuido a disminuir la mortalidad de las complicaciones de la apendicitis. Las modernas técnicas de imágenes, como el ultrasonido y la tomografía computada, también han contribuido a precisar el diagnóstico en los casos difíciles o con una clínica dudosa.

La tendencia contemporánea, desde fines del siglo pasado de ir a la cirugía de menor invasión, también ha involucrado a la cirugía de la apendicitis, cuyos resultados han sido motivo de una controversia menor, que no es tema de este trabajo, pero no parecen ser significativamente diferentes a los de la apendicectomía clásica en la mayoría de los enfermos con una apendicitis simple, no complicada; sí parece ser de mayor utilidad como procedimiento diagnóstico y terapéutico, en especial en la mujer en edad fértil con un cuadro clínico dudoso, como también es de mayor indicación en el paciente obeso. Las primeras apendicectomías electivas por vía laparoscópica fueron comunicadas por Semm ${ }^{20}$ en 1983 y luego la primera serie en apendicitis aguda por Screiber ${ }^{21}$ en 1988. En Chile también se han comunicado, desde hace varios años, experiencias favorables con la apendicectomía laparoscópica ${ }^{22,23}$.

Ahora bien, la Medicina es viva y evoluciona. Como muchos principios en cirugía y en la medicina en general, después de haber sido sancionados, tanto el conocimiento como el tratamiento de una determinada enfermedad, por un consenso, a veces de difícil alcance, como en este caso en que, hace más de un siglo, se acordó con dificultad que la apendicitis aguda era de tratamiento quirúrgico, ahora hay posiciones y estudios que podrían, teóricamente, significar cambios en el enfoque terapéutico de difícil predicción. Así, un meta análisis de reciente publicación demuestra que el tratamiento antibiótico es seguro e igualmente efectivo que la cirugía, como tratamiento primario, de la apendicitis aguda no complicada ${ }^{24}$. Así, según estos autores, el tratamiento antibiótico inicial para el tratamiento precoz de la apendicitis aguda no complicada ameritaría ser considerado. El futuro, como siempre está abierto y lo trabajarán y lo verán los jóvenes cirujanos actuales.

\section{Referencias}

1. Niño Salcedo JA. El cólico miserere y el dolor abdominal. CIRUPED 2012;2:61-3.

2. Fray Gil de Villalón. Del dolor hiliaco o miserere mei. En: Nuevo Thesoro de medicina sacado de los aphorismos de la caridad: según la práctica de muchos enfermeros capuchinos, assi españoles, como italianos. Madrid. Antonio Marín. 1750;pp306.

3. Acea Nebril B. El cólico miserere (Miserere mei). Aportaciones sobre su etimología y características clínicas e hipótesis sobre su aparición en la literatura médica de los siglos XVII-XVIII. Rev Esp Enferm Dig. 2001;93:176-80.

4. Wangesteen $\mathrm{OH}$. Historical Aspects of the management of acute intestinal obstruction. Surgery 1969;65:36383.

5. Surgery. An illustrated History. Rutkow I.M (ed). Mosby-Year Book, Inc. 1933. pp321.

6. Brotóns Barcón J. Pasión ilíaca derecha. Medicina e Historia 1996;62:1-16.

7. García-Sancho L. La apendicitis aguda: su influencia en la historia de la humanidad. Anales de la Real Academia Nacional de Medicina 2006;73:869-90.

8. Williams GR. Presidential Address: A History of Appendicitis. Ann Surg. 1983;197:495-506.

9. De Santo NG, Bisaccia C, De Santo LC, De Santo RM, Di Leo VA, Papalia T, et al. Am J Nephrol. 1999;19:199-212.

10. Meade RH. The evolution of surgery for appendicitis. Surgery 1964;55:741-52.

11. Statti M. Apéndice, tiflitis, apendicitis, apendicectomía, antibióticos y laparoscopía: un largo camino. Revista del Hospital Privado de Comunidad. Mar del Plata, Argentina 2004;7:56-9.

12. Saade RA, Benítez G, Aponte ME. Historia del diagnóstico y tratamiento de la apendicitis aguda. Rev Fac Med Caracas 2005;28:75-8.

13. Barcat JA. Sobre la apendicitis aguda: Amyand, Fitz, y unos pocos más. MEDICINA (Buenos Aires) 2010;70:576-8.

14. Loveland JE. Reginald Heber Fitz, the exponent of appendicitis. Yale Journal of Biology and Medicine 1937;9:509-20.

15. Martínez G, Reyes HE. Charles Heber McBurney: la incision, el punto y el cirujano. Cir Gen. 2007;29:70-5.

16. Morgenstern L. Charles McBurney (1845-1913). A field from the appendix. Surg Endosc. 1996;10:385-6.

17. Mirilas P, Skandalakis JE. Not justa an appendix: Sir Frederick Treves. Arc Dis Child. 2003;88:549-53.

18. Reccius A. Historia y desarrollo de la Cirugía Abdominal en Chile. Ed. Zig-Zag. Santiago de Chile 1948. pp 276.

19. Zúñiga S. Ochenta años atrás: esbozos de la pediatría y la cirugía pediátrica en el país. Rev Chil Pediatr. 2012; 83:420-2. 
20. Semm K. Endoscopic Appendectomy. Endoscopy 1983;15:58-64.

21. Screiber JH. Early experience with laparoscopic appendectomy in women. Surg Endosc. 1987;1:211-6.

22. Vallejos C. Apendicectomía laparoscópica vs apendicectomía abierta en apendicitis aguda. Experiencia del Hospital de Coyhaique. Rev Chil Cir. 2006;58:114-21.
23. Rivera C, Ross M. Apendicectomía laparoscópica en peritonitis apendicular. Rev Chil Cir. 2001;53:186-9.

24. Varadhan K, Neal KR, Lobo DN. Safety and efficacy of antibiotics compared with appendicectomy for treatment of uncomplicated acute appendicitis: meta-analysis of randomized controlled trials. BMJ 2012;344:e2156 doi:10.1136/bmj.e2156 (Published 5 April 2012). 